\title{
Encoding Circuit, Entropy and Error Correction
}

\author{
Subhash Kak
}

\begin{abstract}
This paper considers the entropy perspective on the problem of noise in the circuit where the quantum data is prepared before it is sent forward to the error correction encoder. Since the errors in the circuits before the data qubits are converted to logical qubits cannot be corrected, there will be residual qubit errors in the processing system. This constitutes a great challenge for developing useful, scalable quantum computers.
\end{abstract}

\section{INTRODUCTION}

Classical systems use thresholding to separate bits, and this endows them with the capacity to eliminate small errors that are inevitable in the encoding circuit. We can see this in the case of bounded noise, which causes errors less than half the separation between adjacent levels, which can be readily corrected. But if noise is not constrained as above, there will be residual errors, which in classical error correction coding schemes are corrected by using the redundancy that is distributed over the bits of the coding block.

For data in an information block of size $\mathrm{k}$ bits, which is increased to $\mathrm{n}$ code bits, there are potentially $2^{\mathrm{n}}$ possible binary combinations for each codeword. Since the number of codewords is $2^{k}$, a total of $2^{n k}$ different possible random codes may be imagined. This redundancy makes it possible to correct errors within the sphere associated with each codeword.

Mathematically, the classical situation is that of $Z=X+n$, where $X$ is the input signal, $n$ is noise (not to be confused with $n$, the size of the codeword), $\mathrm{Z}$ is the noisy signal; also let $\mathrm{Y}$ be the output after $\mathrm{Z}$ is passed through an error correction circuit. Assuming that $\mathrm{X}$ and $n$ are independent, the entropy $\mathrm{H}(X, n)=\mathrm{H}(X)+\mathrm{H}(\mathrm{n})$. The correlations between the bits of $\mathrm{X}$ within the encoding block can be used to correct errors within the error-correction capability of the code [1] that assumes certain limits on the nature of noise. Since all errors cannot be corrected in the general case, the entropy $H(Y) \geq H(X)$.

Quantum error correction codes [2][3] are a generalization of the classical ones, but there are crucial differences between them due to the fact that qubits cannot be cloned and one cannot make projective measurements on all the qubits until the computation is complete. To see these differences it helps to investigate the error correction process at the physical level [4] so as to determine the nature of the mapping between the mathematical theory of error correction and the proposed physically realizable components. 
Quantum computation systems assume that the encoding circuit process is error-free which is an idealization [5][6][7]. In these papers it was pointed out that there exist issues regarding loading of information, implicit use of ideal elements, and the problem of scalability [8]. It was also argued that from a thermodynamic perspective a scalable quantum memory system cannot be realized since it can then be used as a perpetual motion machine of the second kind [9].

It has been suggested that a fault tolerant surface code logical qubit will require a lattice with over a thousand qubits [10], when the largest "quantum computers" that have been built have one hundred qubits. Proposals for a practical quantum computer require the control of an astronomically large set of parameters with continuous values, therefore the challenge for building a useful quantum computer is quite daunting [11].

In this paper the issues associated with the encoding circuit used before implementing the actual coding scheme will be reviewed for both classical and quantum error correction coding to determine the exact way these two schemes are different. Specifically, we wish to consider the impact of bounded and unbounded noise and establish some general results.

In the larger scheme, one must also consider additional sources of noise such as those arising from the implicit assumptions about the nature of space and time. Thus it has been proposed that space dimensionality may be a fractal with a value somewhat less than three and some evidence has been adduced for it [12][13][14][15]. There are further issues related to quantum fractals and optimal data representation [16][17][18][19][20].These could have hitherto unknown implications for the computational process in a large circuit and should caution us regarding challenges of implementation in the building of a practical and useful quantum computer.

\section{ENCODING IN CLASSICAL ERROR CORRECTION}

Let us first consider the situation of the encoding circuit in the classical setting. Certain small bounded errors in the circuit hardware do not matter because the physical signal is sampled and after thresholding it can be reconstructed. The system is divided into two parts: the k-to-n expansion that takes the information word to the codeword $(n \geq k)$, and the modulator that puts the digital data on the physical channel as a waveform (Figure 1).

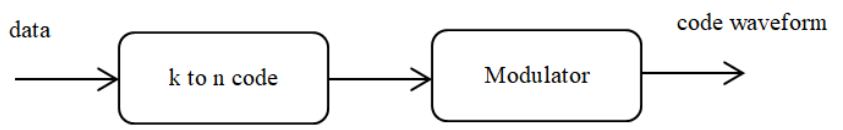

Figure 1. A classical error correcting system

A common method is to let a positive pulse $f(t)$ represent the bit 1 and its flipped form 
$-f(t)$ represent the bit 0 at time $t=0$. Mathematically, the expression for the example bit sequence $S: 101011$ will then be:

$$
S(\mathrm{t})=\mathrm{f}(0)-\mathrm{f}(\mathrm{t}-1)+\mathrm{f}(\mathrm{t}-2)-\mathrm{f}(\mathrm{t}-3)+\mathrm{f}(\mathrm{t}-4)+\mathrm{f}(\mathrm{t}-5)
$$

The bits are put on the channel or the physical hardware using either phase shift or amplitude shift keying (Figure 2, for S: $\left.\begin{array}{llllllllll}0 & 0 & 1 & 0 & 1 & 0 & 1 & 1 & 0 & 0\end{array}\right)$. The correct sequence is recovered back from $S_{n}(t)$ by sampling and checking if the value of the observed signal is greater or less than zero, given the coding of the bits is bipolar: if the sample is less than zero it is mapped to 0 and if it is more than zero it is mapped to 1 .
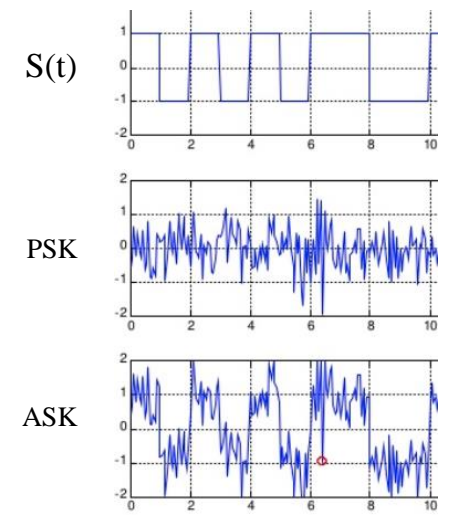

Figure 2. The signal $\mathrm{S}(\mathrm{t})$ and the noisy signal in its phase shift and amplitude shift forms.

Due to the inevitable encoding errors, bandwidth limitations and noise what is generated is the waveform $S_{n}(\mathrm{t})=S(\mathrm{t})+n(\mathrm{t})$, where $n(\mathrm{t})$ is the noise. The entropy at the output will be a sum of the two entropies. The receiver is taken to consist of $2^{k}$ matched filters, one for each codeword, and an error occurs if the matched filter receiver output corresponding to the transmitted codeword is not higher than the other matched filter outputs. We assume that the Hamming distance between the two codewords is $\mathrm{d}$.

For $\mathrm{k}$ information bits, $\mathrm{n}$ code bits are transmitted. If $E_{b}$ is the energy per information bit, then the energy per transmitted bit is $E_{s}=\frac{k E_{b}}{n}$. In the absence of noise, the output of the matched filter receiver for the transmitted codeword is $\mathrm{n} \sqrt{E_{S}}$ and the output of the other codeword matched filter receiver is $(\mathrm{n}-2 \mathrm{~d}) \sqrt{E_{s}}$, where $\mathrm{d}$ is the Hamming distance between codewords [1]. The following results follow easily:

Theorem 1. If noise is bounded and less than $w / 2$, where $w$ is the separation between the bipolar pulses corresponding to the data bits, the probability of decoding error $p_{d}=0$. 
Theorem 2. In the general case, when the noise is white Gaussian with variance per matched filtered received bit equal to $\sigma^{2}=\frac{N_{0}}{2}$, where $N_{0}$ is the single sided noise spectral density, the probability of decoding error is $p_{d}=\frac{1}{2} \operatorname{erfc}\left(\sqrt{d \frac{k}{n} \frac{E_{b}}{N_{0}}}\right)$ where erfc is the complementary error function.

Proof. The residual error is simply the integral of the Gaussian function beyond the radius of the sphere that surrounds each codeword.

Corollary. The error can be made as small as possible by increasing signal energy, the distance between codewords, and the ratio $n / k$.

\section{QUANTUM ENCODING CIRCUIT}

Now consider the quantum information situation. As in the classical case, we break up the error correction system into two parts that are related to the physical processing of information. Figure 3 represents the simple case of a single qubit in an unknown state $|\varphi\rangle=\alpha|0\rangle+\beta|1\rangle$, the first part takes the qubit into a corresponding logical qubit, which is followed by error correction to obtain at the output what was at the input. However, we don't know if the logical qubit does indeed correspond to the input qubit for it cannot be cloned or measured for we don't know what errors may have crept in the encoding circuit.

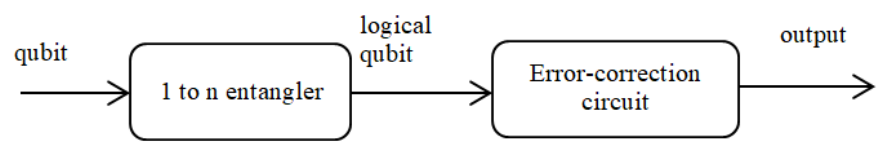

Figure 3. QEC as a black box

The method used to protect the single qubit is by the entanglements within a n-qubit code that creates a logical qubit. For the 3-qubit example, the unknown qubit $|\varphi\rangle=\alpha|0\rangle+\beta|1\rangle$ will be coded into the three qubit state $|\varphi\rangle_{L}=\alpha|000\rangle+\beta|111\rangle$ (as in Figure 4). The simplest quantum error correction method is the application of redundancy quite like how it is used in classical information. For example a triple repetition code $(0 \rightarrow 000$ and $1 \rightarrow 111$ ) in classical information can correct all single errors using majority logic.

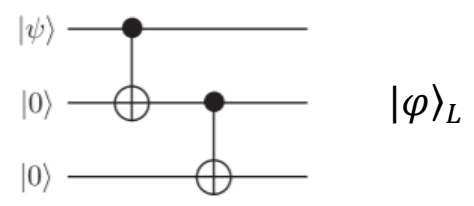

Figure 4. The encoding operation of qubit to logical qubit 
The schematic for the consideration of the logical qubit of Figure 4 and its decoding is given in Figure 5:

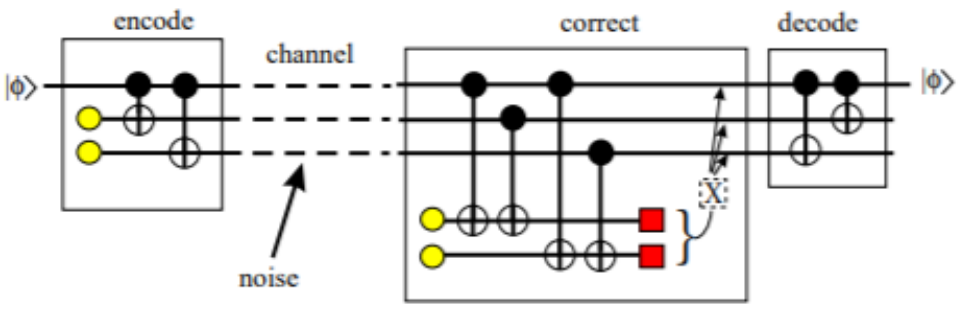

Figure 5. Decoding errors in logical qubit [11]

There are four main blocks that need to be considered: "encode", "channel", "correct", and "decode". The standard quantum error correction theory considers random errors in three of the four blocks but assumes that the "encode" circuit at the beginning of the coding process is ideal, or error-free. If the errors are only in the "channel" and they are within certain bounds, they can be corrected using measurements in the "correct" unit by determining the rotations that are required to obtain the original qubit.

It is assumed Bob receives the three qubits, and they have been acted on by the noise in the channel after error-free encoding. Bob now introduces two qubits of his own, prepared in the state $|00\rangle$. He first carries out controlled-nots from the first and second received qubits to the first ancilla qubit, then from the first and third received qubits to the second ancilla bit. Based on the measurements, appropriately chosen gates are applied on the first qubit to correct it.

In general, errors are analog (continuous in the changes that can occur in the probability amplitudes) [6]. But for simplicity of presentation, we consider a subset of errors that are unitary transformations on the original qubit state and not just bit- or phase-flips. One may now expand the error state in an expansion of the Pauli matrices:

$$
\text { Identity I }=\left[\begin{array}{ll}
1 & 0 \\
0 & 1
\end{array}\right] ; \text { Pauli-X }=\left[\begin{array}{cc}
0 & 1 \\
1 & 0
\end{array}\right], \text { Pauli-Y }=\left[\begin{array}{cc}
0 & -i \\
i & 0
\end{array}\right], \text { Pauli-Z }=\left[\begin{array}{cc}
1 & 0 \\
0 & -1
\end{array}\right]
$$

The single-qubit coherent error may be written as the weighted sum where the $\alpha$ 's are the expansion coefficients:

$$
\alpha_{I}|\varphi\rangle+\alpha_{X} X|\varphi\rangle+\alpha_{Y} Y|\varphi\rangle+\alpha_{Z} Z|\varphi\rangle
$$


Since $\mathrm{Y}=\mathrm{XZ}$ (except for phase), the error may be expressed in the equivalent form:

$$
\alpha_{\mathrm{I}}|\varphi\rangle+\alpha_{\mathrm{X}} \mathrm{X}|\varphi\rangle+\alpha_{\mathrm{XZ}} \mathrm{XZ}|\varphi\rangle+\alpha_{\mathrm{Z}} \mathrm{Z}|\varphi\rangle
$$

Projective measurements are made that cause the above superposition to collapse to a subset of its terms and a code with the ability to correct errors described by the X-and ZPauli matrices will be able to correct any coherent error.

This argument is based on the assumption that the errors occur only in the "channel", after the logical qubit has been constructed. Likewise, it is assumed that no errors occur in the measurements of the "correct" and the "decode" boxes. Both these assumptions are unrealistic and the design of a quantum computer needs to address these sources of error.

The code at the output of the encoding circuit might not be $|\varphi\rangle_{L}=\alpha|000\rangle+\beta|111\rangle$ but a somewhat different $\gamma|000\rangle+\delta|111\rangle$. Since the probability amplitudes of a qubit are continuous values, these values for an unknown qubit entering a non-ideal (noisy) physical system cannot be corrected. This is the key mathematical step that overlooks the possibility of errors in the encoding process. Even if the difference were small for the two sets of complex amplitudes, given many such encodings in a real quantum computer, the effects of this error will be devastating for the computation, and the information about the unknown $\alpha$ and $\beta$ will be irrevocably lost. When there is error in the encoding circuit, the original state $|\varphi\rangle=\alpha|0\rangle+\beta|1\rangle$ cannot be recovered.

Theorem 3. The state at the output of the quantum error-correction circuit cannot be certified as identical to the state entering the encoding circuit.

\section{ENTROPY TRANSFORMATION IN THE BLACK-BOX}

Now we consider the noise in the encoding circuit a bit further. Can there be safeguards within the operations that mitigate the effects of errors? The general error models that have been considered in the literature include bit-flips and phase-flips, as well as "coherent noise" that is modelled by systematic unitary errors. In reality, there will be many different kinds of errors that one must contend with, especially because the quantum states can be reduced by the interaction with the environment. Furthermore, there may be part of the data on the quantum computer that is entangled with the environment. In general, nonlinear noise will be impossible to correct since the underlying framework of quantum mechanics is linear.

Now we consider a very specific noise source in the encoding circuit that increases entropy and fits within the models considered in [21] (Figure 6). 


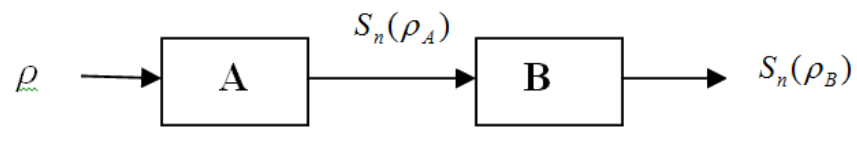

Figure 6. An entropy-increasing form of quantum noise

We assume that A represents data which is qubit $|0\rangle$ and $\mathrm{B}$ is the aggregated effect of the errors within the encoding circuit that injects a qubit $|1\rangle$ with probability $1-\lambda$. The density function of the state created by B will be:

$$
\rho_{B}=\left[\begin{array}{cc}
\lambda & 0 \\
0 & 1-\lambda
\end{array}\right]
$$

This will increase the entropy of the system for $S_{n}(\rho)=-\sum_{i} \lambda_{i} \log \lambda_{i}$, where $\lambda_{i}$ are the eigenvalues of $\rho$. The maximum of the entropy for the binary case will be when $\lambda$ chosen by $\mathrm{B}$ equals $1 / 2$. Even if the value of $\lambda$ is close to 1 , that is the errors are extremely infrequent, the entropy increases. There is nothing within the encoding circuit to counter the increase of entropy, therefore:

Theorem 4. The errors caused by entropy-increasing noise cannot be removed by the errorcorrecting circuit that follows.

\section{A DIALOGUE ON QUANTUM COMPUTING}

The above arguments will now be presented as a dialogue on quantum computing prospects. After Galileo, let's call the proponents of the two main opposing positions Simplicio the physicist and Salviati the engineer, with Sagredo as the neutral layperson who has interest in Mullah Nasruddin stories.

Simplicio: Quantum computing will be one of the key technologies that decide which nation gets to be the leading technological power of the twenty-first century. Quantum computers operate in a mode where several pieces of information are simultaneously processed, making them faster than current-day machines. When built, quantum machines will provide an edge in code-breaking and the solution of certain optimization problems.

\section{Juggling pins on top of an inverted pyramid -}

Salviati: While the mathematics at the basis of quantum algorithms is fine, there are practical engineering reasons why such computers will never be built. 
Unlike a classical computer which uses 0 s and Is (these could be charges or voltages on different points in a circuit), a quantum computer processes states that may be seen as waves together with their phases. Like waves, quantum bits (qubits) exist in superposition.

The quantum waves interact with the environment and the computer can hopelessly decohere in a fraction of a second and so the computation must be completed in very quick time, placing extreme constraints on the system. Without correction of random errors that are inevitable in any physical system the results will be worthless.

Information is physical [21] and the qubits themselves are evolving. One could say that the loading of the information on the quantum computer is like balancing an inverted pyramid, and this balancing is to be done with objects that are themselves flying about in all different directions. The juggler must mount this pyramid and continue juggling the pins while ensuring he doesn't fall down.

Furthermore, a computing machine must correct all small random errors for the computation to be correct. But not all qubit errors can be corrected. Thus errors within the encoder, introduced before the encoding entanglements are introduced, cannot be corrected.

Sagredo: Believe in the future of technology. Remember Archimedes said: Give me a lever and a place to stand, and I shall move the Earth.

Simplicio: NASA, Google, IBM, and other organizations are testing quantum computing systems with around 20 qubits. I know that is far behind the milepost of 50 qubits that was set up by DARPA for 2012. But one must be patient.

Salviati: I read this assessment by Michel Dyakonov in IEEE Spectrum [10]: "There is a tremendous gap between the rudimentary but very hard experiments that have been carried out with a few qubits and the extremely developed quantum-computing theory, which relies on manipulating thousands to millions of qubits to calculate anything useful. That gap is not likely to be closed anytime soon."

He added: "Such a computer would have to be able to manipulate - on a microscopic level and with enormous precision - a physical system characterized by an unimaginably huge set of parameters, each of which can take on a continuous range of values. Could we ever learn to control the more than $10^{300}$ continuously variable parameters defining the quantum state of such a system? My answer is simple. No, never." 
Sagredo: Once Mullah Nasruddin promised his king that he can teach his donkey to read. He said all that he needed was 1,000 dinars and ten years. The king agreed but insisted that if the donkey failed the test, Nasruddin would be put to death. When he reached home with the money, his wife was furious. Relax, Nasruddin told his wife, how does anyone know that a donkey cannot be taught to read. Furthermore, even if a donkey can't be taught remember that ten years is a long time. During that period, the king may be dead, the donkey may be dead, or he may be dead. If they are not dead and if they are unable to teach the donkey, they can flee the kingdom in the tenth year.

Simplicio: Look for the new unexpected research findings that will be obtained as a side result of the quantum computing initiative.

Quantum cryptography --

Simplicio: Quantum cryptography is a breakthrough technology. Two users can exchange keys without anyone able to break the system. Surely, this is a fundamental advance of the greatest importance both for civilian communications and military applications [22][23][24]. And isn't it great that such key exchange can be done using wireless between satellites and with naval ships?

Salviati: But quantum cryptography is used only for key distribution (QKD). First, the two parties must be authenticated, which can only be done classically because the world of conscious agents is classical [25]. Since a chain is only as strong as its weakest link, there is no point doing $\mathrm{QKD}$, for the authentication process itself can be used to exchange keys.

Simplicio: We cannot yield the leadership in this technology to competitors who don't have commitment to democracy and open society.

Sagredo: One day Mullah Nasruddin was in a mischievous mood. He assembled a crowd, and told them a made-up story about gold in the abandoned house beyond the village. When everybody ran to get their hands on the gold, Nasruddin started running with them. When asked by his friends why he was doing so, he said "So many people believe it, that I think it may be true!"

\section{DISCUSSION}

The paper presented an analysis of the encoder that is used prior to the implementation of the error-correction system both for classical and quantum systems.

By assuming that the encoding circuit is error-free, the quantum error correction formalism has implicitly allowed the reference qubit to become available in the framework of the 
latter part of the error correction system. But encoding errors introduced before the quantum error correction process kicks in cannot be corrected and, therefore, pose a challenge to the quantum computer designer. Quantum error correction theory is based on the consideration of the known unknowns and it leaves out the vast world of the unknown unknowns associated with the encoding process.

\section{REFERENCES}

1. R.M. Gray, Entropy and Information Theory. Springer-Verlag, New York (1990)

2. D. Gottesman, An introduction to quantum error correction and fault-tolerant quantum computation. (2009). arXiv:0904.2557

3. J. Roffe, Quantum error correction: an introductory guide. (2019).

4. R. Landauer, The physical nature of information. Physics Letters A 217: 188-193, (1996)

5. S. Kak, The initialization problem in quantum computing. Foundations of Physics 29: 267279 (1999).

6. M.I. Dyakonov, Is fault-tolerant quantum computation really possible? In: Future Trends in Microelectronics. Up the Nano Creek, S. Luryi, J. Xu, and A. Zaslavsky (eds), Wiley, (2007).

7. M.I. Dyakonov, Revisiting the hopes for scalable quantum computation. JETP Letters, v. 98, p. 514 (2013).

8. R. Alicki, Quantum memory as a perpetuum mobile? Stability vs reversibility of information processing. Open Systems \& Information Dynamics, vol.19 no.3, (2012): arXiv:0901.0811

9. A.G. Fowler et al. Surface codes: Towards practical large-scale quantum computation. Physical Review A. 86(3) (2012).

10. M.I. Dyakonov, The case against quantum computing. IEEE Spectrum. 15 November (2018)

11. A.M. Steane, A tutorial on quantum error correction. Proceedings of the International School of Physics "Enrico Fermi”, course CLXII, "Quantum Computers, Algorithms and Chaos”, G. Casati, D. L. Shepelyansky and P. Zoller, eds., pp. 1-32, IOS Press, Amsterdam (2006).

12. S. Kak, Information theory and dimensionality of space. Scientific Reports 10, 20733 (2020).

13. S. Kak, Asymptotic freedom and noninteger dimensionality. Scientific Reports 11, 1-5 (2021).

14. S. Kak, Information theory of evolutionary stages in noninteger dimensional spaces. (2021); https://www.techrxiv.org/articles/preprint/Information_Theory_of_Evolutionary_Stages_in_ Noninteger_Dimensional_Spaces/13775005

15. S. Kak, Fractals with optimal information dimension. Circuits Syst. Signal Process. 40 (2021); https://www.techrxiv.org/articles/preprint/Fractals_with_optimal_information_dimension/133 $\underline{69598}$

16. D. Bercioux, A. Iñiguez, Quantum fractals. Nature Phys 15, 111-112 (2019)

17. J. Li, et al. Scale-invariant magnetic textures in the strongly correlated oxide NdNiO3. Nat Commun 10, 4568 (2019)

18. S. Kak, Logic of representation and information. (2021) https://www.techrxiv.org/articles/preprint/Logic_of_Representation_and_Information/136019 39 
19. S. Kak, The base-e representation of numbers and the power law. Circuits Syst. Signal Process. 40, 490-500 (2021)

20. S. Kak, The intrinsic dimensionality of data. Circuits Syst. Signal Process. 40, 2599-2607 (2021)

21. R. Landuaer, The physical nature of information. Physics Letters A 217, 188-193 (1996)

22. S. Pirandola et al. Advances in quantum cryptography. Adv. Opt. Photon. 12, 1012-1236 (2020)

23. A. Huang et al, Implementation vulnerabilities in general quantum cryptography. New $\mathbf{J}$. Phys. 20103016 (2018)

24. D. Cardinal, Quantum cryptography demystified: How it works in plain language. Extreme Tech. March 11 (2019)

25. S. Kak, State ensembles and quantum entropy. International Journal of Theoretical Physics. 55: 3016-3026 (2016) 